\title{
Modeling and Improving Named Data Networking over IEEE 802.15.4
}

\author{
Amar Abane \\ NIST \\ MD, USA \\ amar.abane@nist.gov
}

\author{
Paul Muhlethaler \\ Inria \\ Paris, France \\ Paul.Muhlethaler@inria.fr
}

\author{
Samia Bouzefrane \\ CNAM \\ Paris, France \\ samia.bouzefrane@lecnam.net
}

\author{
Abdella Battou \\ NIST \\ MD, USA \\ abdella.battou@nist.gov
}

\begin{abstract}
Enabling Named Data Networking (NDN) in realworld Internet of Things (IoT) deployments becomes essential to benefit from Information Centric Networking (ICN) features in current IoT systems. To design realistic NDN-based communication solutions for IoT, revisiting mainstream technologies such as low-power wireless standards may be the key. In this paper, we explore the NDN forwarding over IEEE 802.15.4 by modeling a broadcast-based forwarding strategy. Based on the observations, we adapt the Carrier-Sense Multiple Access (CSMA) algorithm of 802.15.4 to improve NDN wireless forwarding while reducing broadcast effects in terms of packet redundancy, round-trip time and energy consumption.
\end{abstract}

Index Terms-NDN, ICN, IoT, IEEE 802.15.4, Mathematical Model, CSMA, Low-power wireless

\section{INTRODUCTION}

Internet of Things (IoT) systems are built with battery powered devices with limited computation and memory capacity. Device interconnection is achieved with low-rate wireless technologies which allow communication with a satisfactory data rate, payload size and distance range, all with years of battery lifetime. One of these technologies, the IEEE 802.15.4 [1], greatly contributes to make IoT possible. To support IoT solutions with IP, Internet Engineering Task Force (IETF) efforts have resulted in extensions to TCP/IP protocols and the appearance of various other protocols acting like middleware between the application layer and the network layer. Nonetheless, IP still suffers from severe limitations that are becoming difficult to address as they are related to the host-based networking paradigm itself, as studied in [2]. In practice, most IP solutions support the IoT at application level using the Representational State Transfer (REST) architecture, which indicates that the TCP/IP stack has reached its limit to support these new requirements.

Unlike host-based networking, Named Data Networking (NDN) [3] operates with named content. In NDN, every piece of content is identified by a unique name which applications use to request data. Content names are independent from host location; that is, each content item keeps the same name everywhere at producers, caches and consumers. This feature is combined with cryptographic operations to provide self-secured reusable packets and enable in-network caching, since a packet is independent from its source and destination hosts. NDN is an evolved L3 protocol, as it includes some of the relevant operations traditionally provided by higher network layers such as security and flow control. With these features, NDN can match most IoT applications that focus on the content regardless of where it is located or how it is transported. Current efforts regarding NDN for the IoT consist in supporting applications in a simpler, more efficient, and more elegant way [4]. Lightweight design in constrained wireless networks can be considered as a major step towards enabling NDN in low-end IoT. However, in NDN's journey to real-world IoT deployments, we believe that adaptations of current IoT-related technologies will be required. One of these adaptations concerns link-layer technologies, and particularly the 802.15.4 as investigated in this paper.

In this context, we first model a broadcast-based NDN forwarding strategy for wireless constrained networks. The model estimates the average number of frames transmitted per request (i.e., Interest-Data exchange) and the mean roundtrip time (RTT) under content popularity considerations. After that, based on mathematical and experimental observations, we consider an adaptation of the Carrier-Sense Multiple Access (CSMA) algorithm to achieve a trade-off between the Interest satisfaction, the number of transmissions, and the round-trip time. The designed adaptation, Named-Data CSMA (NDCSMA), is derived from the CSMA algorithm to provide a better support for the broadcast-based forwarding approach.

The rest of the paper is organized as follows. Section II gives an overview of basic wireless forwarding in NDN. In Section III, the NDN wireless forwarding model is formulated and evaluated. Section IV describes and evaluates the proposed ND-CSMA scheme. Section V briefly reports on some Information Centric Networking (ICN) and NDN models formulated so far. Section VI concludes the paper with some future perspectives.

\section{Wireless Named Data Networking}

NDN packets do not carry source or destination addresses; packets are forwarded based on their names. Each node maintains two data structures: the Forwarding Information Base (FIB) and the Pending Interest Table (PIT). Optionally, a Content Store (CS) is used to provide in-network caching. In wireless networks, using host addresses to forward packets reduces the data dissemination potential of NDN and limits its benefits. Moreover, mapping names to addresses requires transmission overhead and more memory to maintain 
routes. Therefore, wireless forwarding strategies commonly use broadcast to forward packets without maintaining FIB information. A simple broadcast-based forwarding strategy is the Controlled Flooding (CF) [5]. CF is lightweight and efficient in finding requested content, even under node mobility and intermittent connectivity. CF operates as follows. The communication is initiated by the consumer that requests a content item by sending an Interest. Upon receiving the Interest, an intermediate node first checks if the requested Data locally exists in its CS. If a corresponding Data is found, it is sent back as a response without forwarding the Interest any further. When no matching data is found locally, the router checks the PIT; if an Interest for the same content is already pending, the new Interest is not forwarded. The Interest is forwarded if no similar Interest is already in the PIT. In this case, to avoid redundancy relay-nodes exploit broadcast communications to overhear packets and possibly cancel their transmissions. To do so, every relay-node defers a packet transmission with a random delay during which it keeps listening on the shared wireless medium. While waiting, if the node overhears a packet (i.e., Interest or Data) with the same name, it cancels its transmission. In practice, Interest and Data transmissions are deferred for $\Delta_{I}$ and $\Delta_{D}$ periods of time respectively. Both $\Delta_{I}$ and $\Delta_{D}$ are computed based on an interval, defer window $(d w)$, from which an integer value is randomly chosen to generate the waiting delays as follows [6]:

$\Delta_{D}=\operatorname{rand}[0, d w-1] \times$ DeferSlotTime,

$$
\Delta_{I}=\operatorname{rand}[d w, 2 d w] \times \text { DeferSlotTime, }
$$

where DeferSlotTime is a short period of time.

Here, $\Delta_{I}$ and $\Delta_{D}$ are selected in disjoint intervals with $\Delta_{I}>\Delta_{D}$ to give higher priority to Data packet transmissions.

Once the Interest is forwarded, the relay-node records it in the PIT. When the Interest reaches the content producer or an intermediate cache node, a Data packet containing the requested content is sent back. When the Data reaches a relaynode, it is forwarded if a corresponding Interest exists in the PIT. After that, the router discards the entry from the PIT, and stores a copy of the Data in its CS. The forwarded Data follows the reverse path of the Interest(s) hop-by-hop until it reaches the consumer(s). If a node receives a Data without a matching entry in the PIT, the packet is dropped.

Note that the CF strategy is mainly used with IEEE 802.11 technologies as it requires quite a high bandwidth to achieve good performance. However, CF can be envisioned over 802.15.4 when extremely low latency is not required. Despite deferring transmissions, flooding packets still causes high overhead and packet redundancy. In order to study the applicability of CF over constrained wireless networks, in the next section, we formulate an analytical model of $\mathrm{CF}$ considering content popularity.

\section{Controlled Flooding Model}

\section{A. Assumptions and Notation}

We consider an IoT deployment with consumer applications requesting content produced by wireless devices. Each IoT device is provided with a single 802.15.4 interface and consumers request data through a gateway. The model assumes ideal physical-layer conditions. The network topology is considered as a full binary tree of depth $N$, in which the root and the leaves represent the gateway and the enddevices respectively, and the other nodes represent the relaynodes. Nodes are fixed, sibling nodes can overhear each other, but for the sake of simplicity we assume that no packet is transmitted between them. Consequently, only one path is possible between the gateway and each content producer. Relay-nodes (including the gateway) at the same level have caches of the same size. This means that the cache size is larger in the nodes closer to the gateway, and the gateway has the largest cache size.

The modeled metrics are the following: (i) Cost-per-request (CPR). The number of packets transmitted in the network to retrieve some content requested from the gateway. (ii) Roundtrip time per request (RPR). The mean delay time (in $m s$ ) measured by the gateway from sending an Interest to receiving a matching Data.

\section{B. Content Popularity}

Each relay-node in the network has a cache managed with the Least Recently Used (LRU) replacement policy. We consider a set of $M$ content items equally divided into $K$ classes, each one containing $m=M / K$ content items. Each class represents a different popularity to be requested with probability $q_{k}, k=1,2, \ldots, K$.

The content requested in our scenario can be considered as Web content which usually follows Zipf distribution [7]. Hence, to model the popularity of content classes we use a Zipf distribution, $q_{k}=c / k^{\alpha}$ with $\alpha>1$ and $c=1 / \sum_{k=1}^{K} 1 / k^{\alpha}$. As content in the modeled application consists of a small amount of data, we consider that each content item is transmitted in one Data packet. We assume that each end-device produces the same number of content items of each class, and an item can not be produced by two different devices. The Interest arrival process is modeled through a Markov Modulated Rate Process (MMRP) of intensity $\lambda$. Interests for content in class $k$ are generated according to a Poisson process of intensity $\lambda_{k}=\lambda q_{k}$, and the requested content within the class is uniformly chosen among the $m$ different content items in the given class. That is, a given item in class $k$ is requested with probability $q_{k} / m$. The notations and their meanings are summarized in Table I.

\section{Model Formulation}

We start by defining $p_{k}, p_{k}(i)$ and $p_{t}$. According to the topological and content production assumptions, there is no data duplication among caches at the same level. This allows us to consider all the caches at each level $i$ simply as one cache. Given this, at the first level (i.e., gateway), the stationary 
TABLE I: Model variables

\begin{tabular}{|c|c|}
\hline Notation & Meaning \\
\hline$p_{k}, p_{k}(i)$ & Cache miss probability for class $k$ content \\
& at the gateway, at level $i>1$. \\
$p_{t}$ & Probability that two sibling nodes transmit the same Interest \\
$N$ & given that a cache miss has occurred in both nodes. \\
$K$ & Tree depth. \\
$M$ & Number of popularity classes. \\
$x$ & Number of total content items $(m=M / K$ in each class $k$ ). \\
$\lambda, \lambda(i)$ & Cache size in each level in number of chunks. \\
$\lambda_{k}$ & Content request rate at the gateway, at level $i>1$. \\
$\sigma$ & Content request rate at the gateway for class $k$. \\
$q_{k}, q_{k}(i)$ & Average content size in number of chunks. \\
$d w$ & Content popularity distribution of requests \\
$r_{I}$ & at the gateway, at level $i$. \\
$r_{D}$ & Defer window. \\
$\tau$ & Time needed to send an Interest over one hop (excluding waiting delays). \\
$p_{f}$ & Time needed to send a Data over one hop (excluding waiting delays). \\
\multicolumn{2}{c}{ Pefer slot-time. } \\
\hline
\end{tabular}

miss popularity for chunks of class $k, p_{k}$ is defined and proven in [8] as follows:

$$
p_{k} \equiv p_{k}(1) \approx \exp ^{-\frac{\lambda}{m} q_{k} g x^{\alpha}}
$$

for relatively large $x$, where $1 / g=\lambda c \sigma^{\alpha} m^{\alpha-1} \Gamma\left(1-\frac{1}{\alpha}\right)^{\alpha}$.

Considering a binary tree with $N$ levels and an MMRP content request process with rate $\lambda(i)$, under the popularity distribution given above, the miss probability at level $i \in$ $[2, N)$ is also defined and proven in [8] as follows:

$$
\log p_{k}(i)=\log p_{k}(1) \prod_{l=1}^{i-1} p_{k}(l) .
$$

For more details on Equations 3 and 4, including proof and discussions, readers may refer to [8].

When a cache miss occurs at two sibling nodes, they will both try to forward the Interest after a random delay, as described in Section II. Given the delays computed in Equation 1 , the same Interest may be forwarded by both nodes if they choose random numbers with a difference smaller than $s=r_{I} / \tau$. Hence, the probability that two sibling nodes transmit the same Interest is equivalent to the probability that two random numbers chosen from the interval of length $S=d w+1$ have a difference smaller than $s$. This can be formulated as follows:

$$
p_{t}=1-\left(\left(\frac{S-2 s}{S}\right)^{2}+2 \sum_{i=0}^{s-1}\left(\frac{S-(i+s)}{S^{2}}\right)\right) .
$$

Here, we can define the CPR for retrieving a class $k$ content item as follows:

$$
C P R_{k}=\sum_{i=1}^{N}\left(\left(1-p_{k}(i)\right) \prod_{j=1}^{i-1} p_{k}(j)\right) \times\left(2(i-1)+\prod_{l=2}^{i-1} p_{t} C_{k}(l)\right),
$$

where:

$$
C_{k}(l)=1+p_{f}\left((N-l-1)+\prod_{n=l+1}^{N-1} p_{t} C_{k}(n)\right) .
$$

Note that Equation 6 models the CPR only for the requests that have been satisfied. That is, $p_{k}(N)=0$.

Equation 6 is obtained based on the following approach. As the content can be found at any level from 1 to $N$, the cost is defined as a weighted sum of the transmitted packets associated to each level $i$. The weights correspond to the cache hit probability $\left(1-p_{k}(i)\right)$ at level $i$ given that a cache miss occurred at all the previous levels.

For every possible level $i$, the number of packets is composed of two parts: $2(i-1)$ corresponds to the number of packets transmitted along the path from the gateway to the level- $i$ device, plus the number of packets transmitted if the sibling of each previous node (from level 2 to $i-1$ ) has also transmitted the Interest, which has a probability $p_{t}$ of occurring for each pair of siblings.

Here, Equation 7 assumes that when the brother of a node (at level $l$ ) transmits an Interest, the cost can be recursively computed using the same approach as Equation 6 in its subtree (from level $l+1$ to $N$ ). The only difference is that, on this side of the network, we directly consider the path from level $l$ to the leaf level $N$, since the requested content has already been found elsewhere and there is no data duplication. However, in each sub-tree, the first Interest is always transmitted, but the number of transmissions recursively computed is subject to the probability that no collision occurs between the first sibling nodes of the sub-tree $\left(p_{f}\right)$.

Following the same approach, we define the mean RPR for a class $k$ content item as follows:

$$
R P R_{k}=\sum_{i=1}^{N}\left(\left(1-p_{k}(i)\right) \prod_{j=1}^{i-1} p_{k}(i)\right) R_{i},
$$

where $R_{i}=(i-1)\left(r_{I}+r_{D}+\delta_{I}+\delta_{D}\right)$. Here, $R_{i}$ is obtained by multiplying the number of hops $(i-1)$ for level $i$ by the total delay needed to send an Interest and get Data; which includes waiting delays $\left(\delta_{I}+\delta_{D}\right)$ and time-on-air $\left(r_{I}+r_{D}\right)$.

When two sibling nodes delay their transmissions, the node with the shortest delay will transmit the packet first. Furthermore, the round-trip delay measured by the consumer (e.g., gateway) will be affected by the shortest waiting delay computed at each level. Hence, the global estimation of $\delta_{I}$ and $\delta_{D}$ is not the half way between the lowest and the highest values (e.g., $d w / 2 \tau$ ). To approximate the values of $\delta_{I}$ and $\delta_{D}$, we consider the mean of the lowest half of $[0, d w-1]$ and $[d w, 2 d w]$ intervals respectively. This gives us $\delta_{I}=((3 d w) / 4) \tau$ and $\delta_{D}=((d w-1) / 4) \tau$.

\section{Model Evaluation}

We assess the accuracy of our model using the CF strategy provided by the NDN simulation framework for OMNeT++ [9]. We consider a tree of depth $N=4$. The gateway requests content from a total of $M=3000$ items, distributed in $K=$ 50 classes of decreasing popularity, each one with $m=60$ items. The request rate at the gateway is $\lambda=1$ request/s. We set up a cache of size $x=300$ packets at each level of the tree. Different popularity distributions have been simulated with $\alpha \in\{1.5,2,2.5\}$. Each simulation result corresponds to a run of 10 hours. Each Interest packet has a size of 30 bytes and each Data packet 90 bytes. Preliminary simulations have been used to set optimal values at $d w=127$ and $\tau=0.032 \mu \mathrm{s}$. We also measured $r_{I}=1.36 \mathrm{~ms}, r_{D}=3 r_{I}$ and $p_{f}=0.8$ with preliminary simulations.

First, the Interest satisfaction rate is reported in Table II. We observe that $d w=127$ always achieves better Interest 
TABLE II: Interest satisfaction rate

\begin{tabular}{|c|c|c|}
\hline & $d w=127$ & $d w=255$ \\
\hline$\alpha=1.5$ & $87.8 \%$ & $84.1 \%$ \\
\hline$\alpha=2.0$ & $95.1 \%$ & $93.2 \%$ \\
\hline$\alpha=2.5$ & $98.2 \%$ & $97.4 \%$ \\
\hline
\end{tabular}

satisfaction rate than 255 . The reason is that 127 is low enough to make relay nodes transmit more packets and explore the network without being too low to create a lot of collisions. However, the remaining results show that this Interest satisfaction rate is achieved at the cost of much more transmissions than $d w=255$.

Figures $1 \mathrm{a}, 1 \mathrm{~b}$, and $1 \mathrm{c}$ show the CPR according to content popularity for $\alpha=1.5,2$ and 2.5 respectively. According to the results, the value of $\alpha$ has an impact on the efficiency of NDN. In fact, small values of $\alpha$ reduce popularity difference between classes, which introduces more diversity in the requests and thus increases the cache miss rate and CPR.

The model is also affected by $\alpha$ but can accurately predict the CPR according to popularity classes. The highest discrepancies between the model and simulations are observed for higher values of $\alpha$. The reason is that a cache miss is more likely to occur when $\alpha$ is higher, which leads to more transmissions. Since $d w=127$ is not high enough to avoid all redundancies, the behavior of the nodes becomes more dependent on the link-layer, which is not included in the model.

As a reference, we represent the CPR for a perfect-unicast scenario, which refers to the CPR expected in the tree if a host-based routing protocol is used instead of NDN. We note that NDN with CF outperforms perfect-unicast concerning the most popular content. This shows that transmission overhead induced by broadcast can be attenuated by small caches in the presence of popular content. Moreover, this attenuation can outperform the unicast communication pattern, which is here theoretical as it does not include route discovery/maintenance cost.

Figures 1d, 1e, and 1f report on the mean RPR for $\alpha=1.5$, 2 and 2.5 respectively. The same type of observations can be made as for CPR, but a greater dissimilarity is observed between the model and simulations. The reasons are the same as for CPR, with an additional fact related to medium access time. As $d w$ is not high enough to avoid redundant packet transmissions, the link-layer has to resolve more medium access contentions, leading to less accuracy in our model. This can be confirmed by observing raw simulation results (i.e., blue dots) which present higher scatter as $\alpha$ gets higher.

Figures $1 \mathrm{~g}$ and $1 \mathrm{~h}$ show the CPR and RPR respectively for $\alpha=2$ with $d w=255$. We observe that CPR becomes better when a higher value of $d w$ is used. Compared to $d w=127$, up to two transmissions per request are saved for the least popular content when using $d w=255$. This makes NDN even more efficient than the host-based (unicast) approach. However, this comes at the cost of a higher RPR since waiting delays also increase when $d w$ is higher. With $d w=255$, an increase of
$15 \mathrm{~ms}$ of round-trip delay per request is observed for the least popular content then whith $d w=127$.

Overall, we find that a trade-off between cost and roundtrip delays is difficult to achieve with the CF mechanism. On the one hand, trying to reduce waiting delays by reducing $d w$ increases the number of transmissions and collisions as nodes do not have enough time to listen to each other. On the other hand, reducing cost with higher values of $d w$ will induce higher waiting delays. Moreover, nodes are still listening to transmissions when waiting, which is not helpful for energy consumption.

An ideal improvement one may look for is to reduce the round-trip time and energy consumption by eliminating waiting delays while keeping the lowest number of frame transmissions. Since eliminating waiting delays will significantly impact the CPR, we have to question whether a tradeoff theoretically exists that may achieve reasonable CPR, low RTT and a reasonable Interest satisfaction rate. According to the CF strategy, the tree is explored depending on whether both sibling-nodes forward the Interest or only one of them does. In the model evaluation, we find that the best performance for $\mathrm{CF}$ is achieved with $d w=127$. Let the corresponding forwarding probability for each sibling-node pair be $p_{t}^{*}$. Then, we can easily observe that there is no value of $p_{t}$ lower than $p_{t}^{*}$ that can achieve the same or better satisfaction rate, which is confirmed by Table II. That is, a compromise at L3 level that achieves optimal performance is not possible in our configuration.

We believe that this is due to the fact that in the forwarding decision of a node, only the sibling-node is involved. Therefore, by shifting the transmission decision to the L2 level (with some modifications) instead of using deferred transmissions, one may expect better performances since the CSMA algorithm natively considers multiple-access contention. The design and evaluation of our L2-based approach are presented in the next section.

\section{A NAMED-DATA CSMA FOR IEEE 802.15.4}

To eliminate waiting delays without increasing unnecessary transmissions, we replace the waiting delays by a prioritybased CSMA scheme designed for NDN, as described below.

\section{A. Named-Data CSMA Design}

The unslotted CSMA algorithm works with a set of default parameters, and a node maintains two values when transmitting a frame: Number of Back-offs (NB) is the number of access attempts for the current transmission. Back-off Exponent (BE) is used to compute the random back-off period to wait before attempting to assess the channel.

In legacy CSMA, all nodes access the shared channel with a fair chance. Priority-based CSMA [10] uses the difference in traffic type to introduce differentiated channel access for nodes. Therefore, the priority-based CSMA mechanism is designed to make nodes with high priority traffic have a greater chance of accessing the channel. In ND-CSMA, the frames are classified into two priority classes: (i) frames that contain a 


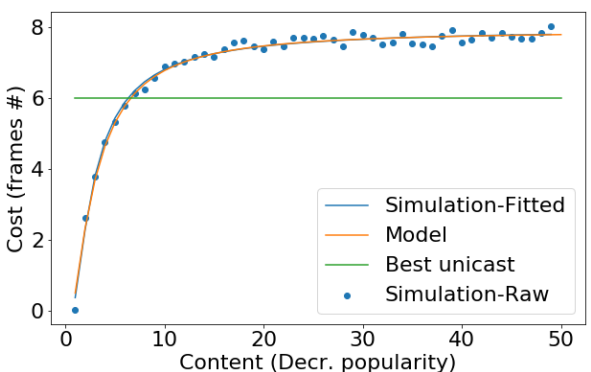

(a) CPR: $d w=127, \alpha=1.5$

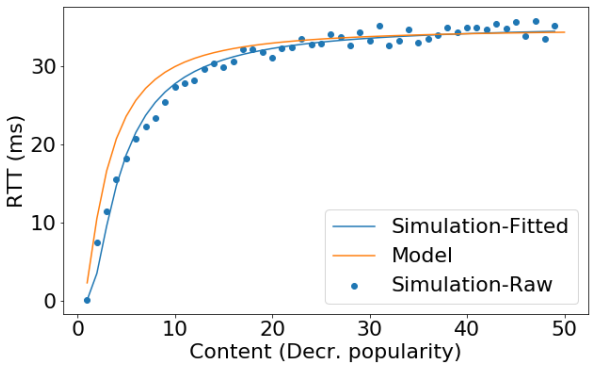

(d) RPR: $d w=127, \alpha=1.5$

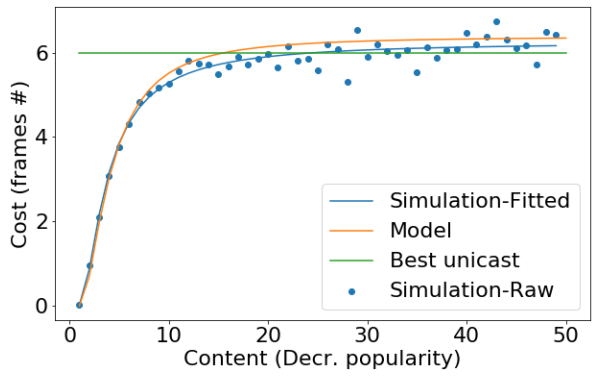

(g) CPR: $d w=255, \alpha=2$

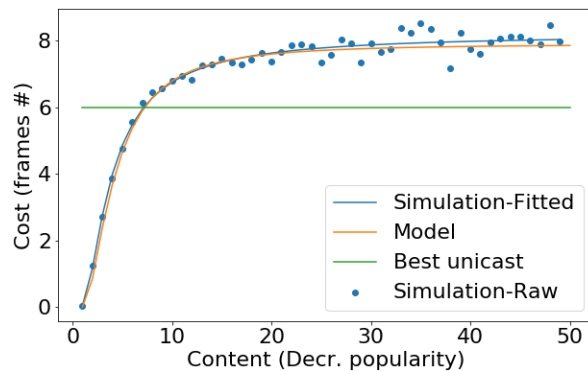

(b) CPR: $d w=127, \alpha=2$

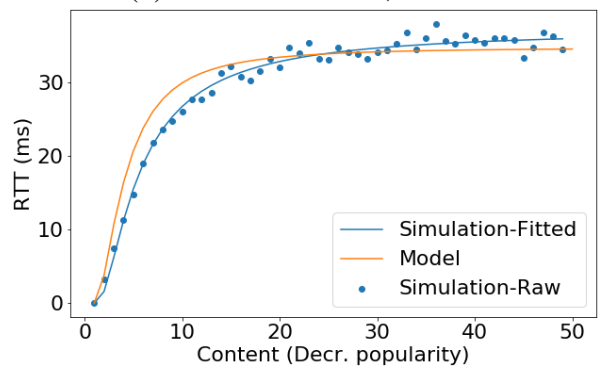

(e) RPR: $d w=127, \alpha=2$

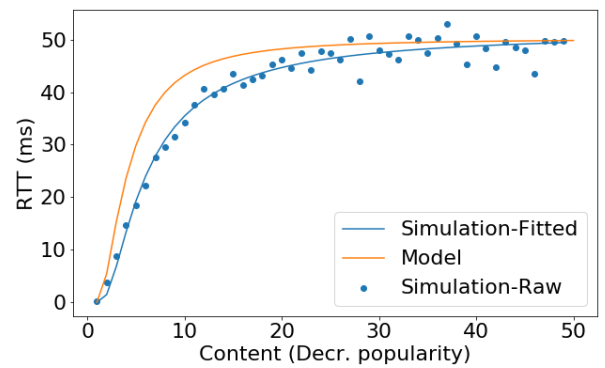

(h) RPR: $d w=255, \alpha=2$

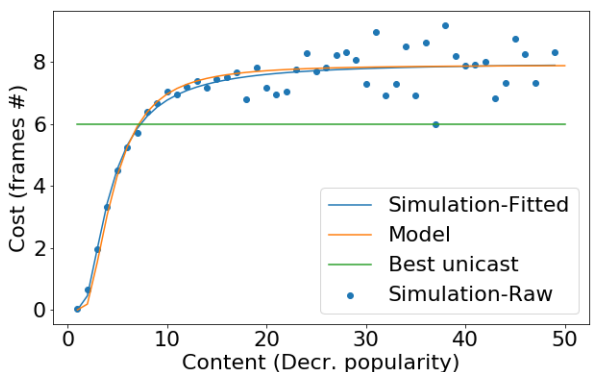

(c) CPR: $d w=127, \alpha=2.5$

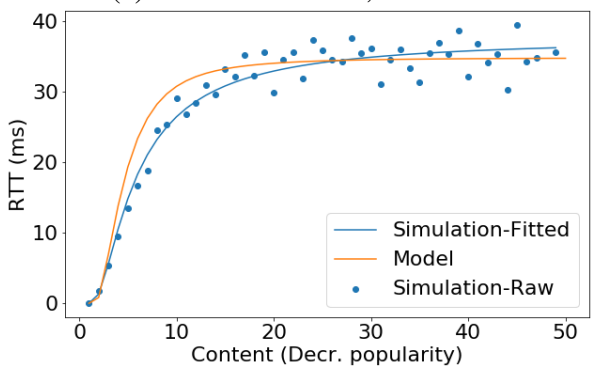

(f) RPR: $d w=127, \alpha=2.5$

Fig. 1: Model evaluation results

Data at any node, and frames that contain a locally issued Interest are assigned a priority 0 . (ii) frames that contain an Interest to forward are assigned a priority 1 . The other parameters are kept the same as in legacy CSMA.

ND-CSMA operate as follows: Step 1. The values of $N B$ and $B E$ are initialized to $O$ and 3 respectively. Step 2. A delay is computed based on a random back-off in the range $\left[0,2^{B E-1}\right]$. Step 3. After the waiting time, the node performs a Clear Channel Assessment. If the channel is idle, the node starts transmission. If the channel is busy, $N B$ is incremented by 1 . Retrying another back-off depends on the priority of the frame. If the frame has a priority 0 , Step 4 is performed (i.e., equivalent to legacy CSMA). If the frame has a priority 1 , the transmission is canceled if $N B \geq t h$, otherwise Step 4 is performed. Step 4. $B E=\min (B E+1, a M a x B E)$, another attempt is performed if $N B<\max C S M A B$ ackoffs, the transmission is canceled otherwise. ${ }^{1}$

\section{B. Evaluation}

To evaluate the ND-CSMA scheme, we simulate three scenarios: (i) CF. The CF strategy with the legacy CSMA. (ii)

\footnotetext{
${ }^{1}$ maxCSMABackoffs and $a M a x B E$ as defined in the standard.
}

BF. The CF strategy without waiting delays, using the legacy CSMA. (iii) ND-CSMA-x. The CF strategy without waiting delays, using ND-CSMA with $t h=x$.

All the scenarios are simulated under the same parameters (i.e., $\mathrm{N}, \mathrm{M}, \mathrm{K}, \mathrm{m}, \mathrm{x}$ ) as those used in Section III. We set $\alpha=2.0$. For the CF strategy, we use $d w=127$ and $\tau=$ $0.032 \mu \mathrm{s}$. We measure the following metrics: (i) RPR. The mean time needed for the gateway to retrieve a content item from a device. (ii) Transmitted frames. The total number of frames successfully transmitted. (iii) Interest satisfaction rate. This corresponds to the number of Data received by the gateway over the number of Interests it sent. (iv) Mean backoff time. The average time the nodes spent in back-off to access the wireless medium.

Figure 2 shows the results obtained. We observe that NDCSMA with th $=1$ achieves the lowest RPR compared to scenarios (i) and (ii). The mean back-off time with NDCSMA-1 is the smallest among the evaluated scenarios, while (ii) achieves the highest back-off time due to the large number of forwarding decisions generated after eliminating waiting delays. That means, legacy CSMA without delays has to resolve medium access contention with more back-off periods 

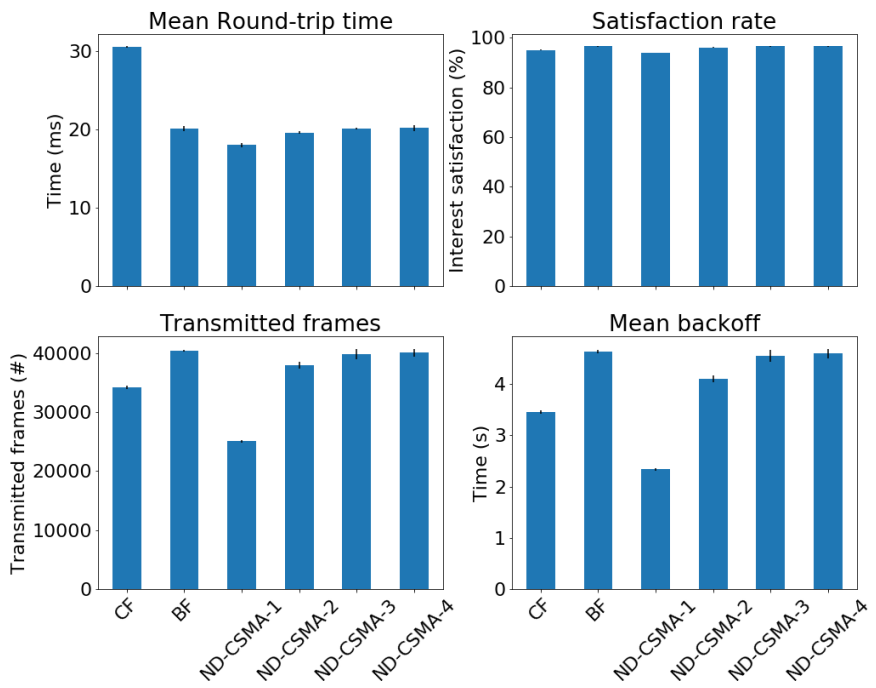

Fig. 2: ND-CSMA evaluation

whereas ND-CSMA has the possibility to cancel some Interest transmissions when the channel is busy rather than waiting for other back-off periods. For this reason, the RPR achieved by scenario (ii) is slightly higher than ND-CSMA. CF also is capable of canceling scheduled transmissions using deferred transmissions. However, it achieves that with a wait-and-listen mechanism which induces higher round-trip delays, and a relatively high back-off time is required when the chosen random delays are not different enough.

Moreover, the results show that forwarding with NDCSMA-1 can ensure necessary packet transmissions while keeping the total cost at a minimum compared to the two other schemes. The Interest satisfaction rate is quite similar for all the approaches, which indicates that ND-CSMA, even with low th does not reduce the efficiency of Interest flooding/broadcast. Furthermore, simulations with small caches (e.g., tens of packets) achieved approximately the same results, but with a slightly lower satisfaction rate for ND-CSMA.

Overall, ND-CSMA-1 seems to be the best compromise for the measured metrics. To return to our theoretical expectations, the results confirm that a link-layer adaptation is able to keep the benefits of a broadcast-based forwarding strategy in terms of satisfaction rate, while reducing medium access contention and the number of transmissions; it then achieves the trade-off we were looking for.

\section{RELATED WORK}

Most of the models on ICN/NDN are conducted exclusively around caching, such as cache deployment, cache decision and cache replacement. Other studies are devoted to ICN/NDN transport and routing performance, often with comparison to TCP/IP, such as in [11]. However, very few analytical models for ICN/NDN consider modeling networks of caches and the interaction between caching and transport; those models consider traditional wired networks [12]. To the best of our knowledge, no model has been formulated on NDN in wireless networks with cache consideration, whether for constrained or traditional wireless networks. We also believe that no previous proposals have been made to adapt the CSMA scheme of the 802.15.4 link-layer to improve NDN wireless forwarding.

\section{CONCLUSiOn AND Future Work}

In this paper, we modeled a simple broadcast-based NDN wireless forwarding strategy. One objective of the model is to show that caching can attenuate the number of transmissions generated by broadcast to achieve a reasonable overhead while keeping the data dissemination power of NDN. Based on a simple modification of the 802.15.4 MAC layer, preliminary results shed light on the necessity of rethinking typical linklayer schemes for ICN/NDN such as the CSMA algorithm. As future work, we aim to explore more complex CSMA adaptations for lightweight forwarding to take the most of NDN and design a general-purpose Named-Data CSMA.

\section{REFERENCES}

[1] IEEE, "Ieee standard for local and metropolitan area networks-part 15.4: Low-rate wireless personal area networks (lr-wpans)," IEEE Std 802.15.4-2011 (Revision of IEEE Std 802.15.4-2006), pp. 1-314, Sep. 2011.

[2] W. Shang, Y. Yu, R. Droms, and L. Zhang, "Challenges in IoT networking via TCP/IP architecture," NDN, Tech. Rep. NDN-0038, February 2016.

[3] L. Zhang, A. Afanasyev, J. Burke, V. Jacobson, kc claffy, P. Crowley, C. Papadopoulos, L. Wang, and B. Zhang, "Named Data Networking," ACM SIG-COMM Computer Communication Review, vol. 44, no. 3, pp. 66-77, July 2014.

[4] W. Shang, A. Bannisy, T. Liangz, Z. Wangx, Y. Yu, A. Afanasyev, J. Thompsonx, J. Burkex, B. Zhangz, and L. Zhang, "Named Data Networking of Things (Invited paper)," in The 1st IEEE Intl. Conf. on Internet-of-Things Design and Implementation, Berlin, Germany, April 2016.

[5] L. Wang, A. Afanasyev, R. Kuntz, R. Vuyyuru, R. Wakikawa, and L. Zhang, "Rapid traffic information dissemination using named data," in Proceedings of the 1st ACM Workshop on Emerging Name-Oriented Mobile Networking Design - Architecture, Algorithms, and Applications, ser. NoM '12. New York, NY, USA: ACM, 2012, pp. 7-12.

[6] M. Amadeo, C. Campolo, and A. Molinaro, "Forwarding strategies in named data wireless ad hoc networks: Design and evaluation," Journal of Network and Computer Applications, vol. 50, no. Supplement C, pp. $148-158,2015$.

[7] L. Breslau, Pei Cao, Li Fan, G. Phillips, and S. Shenker, "Web caching and zipf-like distributions: evidence and implications," in IEEE INFOCOM '99. Conference on Computer Communications. Proceedings. Eighteenth Annual Joint Conference of the IEEE Computer and Communications Societies. The Future is Now (Cat. No.99CH36320), vol. 1, March 1999, pp. 126-134 vol.1.

[8] G. Carofiglio, M. Gallo, L. Muscariello, and D. Perino, "Modeling data transfer in content-centric networking," in 2011 23rd International Teletraffic Congress (ITC), Sep. 2011, pp. 111-118.

[9] A. Abane, P. Muhlethaler, S. Bouzefrane, M. Daoui, and A. Battou, "Towards evaluating named data networking for the iot: A framework for omnet++," 092018.

[10] L. ZHAO, G. wei BAI, H. SHEN, and Z. min TANG, "Priority-based ieee $802.15 .4 \mathrm{csma} / \mathrm{ca}$ mechanism for wsns," The Journal of China Universities of Posts and Telecommunications, vol. 20, no. 1, pp. 47 $-53,2013$.

[11] G. Carofiglio, G. Morabito, L. Muscariello, I. Solis, and M. Varvello, "From content delivery today to information centric networking," Computer Networks, vol. 57, no. 16, pp. 3116 - 3127, 2013, information Centric Networking.

[12] Y. Ren, J. Li, L. Li, S. Shi, J. Zhi, and H. Wu, "Modeling content transfer performance in information-centric networking," Future Generation Computer Systems, vol. 74, pp. 12 - 19, 2017. 